\title{
PLANTAS TEXTILES DE LOS GUARANÍES DE MISIONES, ARGENTINA
}

\author{
HÉCTOR A. KELLER ${ }^{1}$
}

\begin{abstract}
Summary: Keller, H. A. 2009. Textile plants of the Guarani from Misiones, Argentina. Bonplandia 18 (1): 29-37. ISSN: 0524-0476.

Information about textile plants used or mentioned in Guarani communities of Misiones Province, Argentina is here given. Methods of harvest and uses of same plants are detailed. The species are classified in three categories; used frequently at present, used occasionally and used at the past. The manuscript is illustrated by mean photographs.
\end{abstract}

Key words: fibers, ropes, Mbya, Ava Chiripa, Guarani communities.

Resumen: Keller, H. A. 2009. Plantas textiles de los Guaraníes de Misiones, Argentina. Bonplandia 18 (1): 29-37. ISSN: 0524-0476.

Se presenta información sobre las especies de plantas textiles mencionadas o usadas en comunidades guaraníes de la provincia de Misiones, Argentina. Se brindan algunos detalles sobre técnicas de recolección y aprovechamiento de dichas especies. Se especifican las especies que se siguen usando con frecuencia, las que se usan raramente y las que han caído en desuso. Se ilustra el trabajo mediante fotografías.

Palabras clave: fibras, cordeles, Mbya, Ava Chiripa, comunidades guaraníes.

\section{Introducción}

El objetivo de este trabajo es documentar las plantas textiles que utilizan o conocen los integrantes de aldeas guaraníes de la provincia de Misiones, Argentina. Las comunidades entrevistadas se encuentran establecidas en los departamentos Concepción, Eldorado, Guaraní, Libertador General San Martín, Montecarlo y San Pedro; están constituidas en su mayor parte por miembros de la parcialidad Mbya con algunos integrantes Ava Chiripa. La etnografía ha abordado intensamente estos grupos a lo largo de toda su área de dispersión, Paraguay oriental, nordeste de Ar- gentina y sur de Brasil. Como resultado, se han escrito numerosas contribuciones que describen detalladamente sus características culturales. Ya en 1914 Nimuendajú señalaba que sería superfluo continuar escribiendo sobre los guaraníes, sin embargo las contribuciones recientes son también muy abundantes; Meliá (2005) ofrece una reseña donde se nombran a muchos autores que han abordado a este grupo en los últimos cuarenta años.

Diversas actividades tradicionales de los guaraníes, tales como la caza con trampas, la pesca, la recolección, entre otras, han permitido la subsistencia del conocimiento relativo al uso de recursos naturales. En cambio la adopción de prácticas y elementos foráneos lo ha

${ }^{1}$ Instituto de Botánica del Nordeste, Casilla de Correo 209, 3400 Corrientes, Argentina. E-mail: kellerhector@hotmail.com 
deteriorado. El empleo de fibras textiles constituye un ejemplo de este proceso erosivo.

La Real Academia Española define "textil" como "perteneciente o relativo a los tejidos". Como adjetivo se aplica a la materia susceptible de ser reducida a hilos y tejida. Se define planta textil como aquella que produce fibras hilables, siendo el hilo una hebra larga y fina que se obtiene retorciendo cualquier material textil. Sobre la base de estas definiciones, en este trabajo se incluyen las plantas utilizadas por los guaraníes para obtener hilos o cuerdas, así como también aquellas de las cuales se obtienen materiales que, sin ser transformados en hilos, se utilizan para elaborar cestería u otro tipo de manufacturas entretejidas (ej.: hojas y fragmentos de tallos).

Muchas de las especies textiles tradicionales de los guaraníes han caído en desuso debido a la adquisición de materiales sintéticos modernos. Igualmente se incluye en este manuscrito información sobre aquellas plantas cuya identidad y técnica de utilización son aún conocidas, especialmente por las personas mayores.

\section{Material y Métodos}

El trabajo de campo se efectuó durante los años 1998-2008 en doce comunidades guaraníes, en el marco de un relevamiento etnobotánico, con períodos más o menos prolongados de permanencia en las aldeas. Se hicieron entrevistas a la mayor cantidad posible de personas, de diferente sexo y rango etario. Ello permitió detectar a las personas más o menos vinculadas con cada categoría de uso, y profundizar en temas específicos de investigación. Se aplicó la observación participante durante la elaboración cotidiana de diversos productos de la cultura material de cada comunidad. Para el caso de algunas manufacturas textiles que han perdido vigencia, se solicitó la colaboración de ancianos para recrear el proceso de confección de dichos productos. En la tabla del apéndice, se discriminan las plantas que son usadas actualmente con frecuencia (A), las que en la actualidad se utilizan sólo esporádicamente es decir no to- dos los años (E), y las que se mencionan cómo de uso pretérito (P).

Se recolectó material de herbario con el fin de identificar las especies empleadas, dicho material se halla depositado en el herbario CTES del Instituto de Botánica del Nordeste, Corrientes, Argentina.

\section{Resultados y Discusión}

El vocablo guaraní que define las fibras de aplicación textil es "yvi”, y se emplea como sufijo del nombre de la planta que las provee, por ejemplo "pindoryvi" (fibras de las vainas foliares de la palmera "pindo"), pynoryvi (fibras de la corteza de la urticácea Urera baccifera). Dado que "yvi” es también el nombre aplicado a Ceiba speciosa (Bombacaceae), podemos conjeturar que dicho árbol ha representado para los guaraníes la especie textil por antonomasia. Se ha sugerido que los nombres más antiguos de plantas son términos simples que usualmente hacen referencia a su aplicación práctica (Lange, 1966), por esta razón, aunque su uso es actualmente casi nulo, probablemente dicho árbol ha sido una de las primeras especies utilizadas por estos grupos con fines textiles. Otras especies poseen nombres derivativos del vocablo “yvi”, por ejemplo Bromelia balansae y otras bromeliáceas llamadas "yvira" (proveedor de fibras textiles). Aunque no sean usadas actualmente, ni mencionadas como textiles del pasado, algunas especies arbóreas con corteza fibrosa han sido presumiblemente empleadas para este fin, debido a la conformación de sus nombres guaraníes, por ejemplo las borragináceas Patagonula americana o "guajayvi" (textil de los indígenas Guaja) y Cordia trichotoma o “apeteryvi” (corteza textil).

En la tabla del apéndice, se listan un total de 30 especies textiles mencionadas por los guaraníes de Misiones, dichas especies se hallan distribuidas en 15 familias. De este total de especies, 16 (56\%) se utilizan con frecuencia, 4 (13\%) se utilizan esporádicamente y 10 (33\%) ha caído en desuso. Se detallan en dicha tabla, el uso, la parte usada, los nombres 
guaraníes que reciben y un ejemplar de referencia.

Sobre la base de algunas de las especies listadas en la tabla se describen a continuación las principales aplicaciones relativas a las plantas textiles.

\section{Ataduras}

El uso de plantas textiles generalmente exige una secuencia de procedimientos y métodos para la obtención y acondicionamiento del material fibroso. Sin embargo, entre los guaraníes, se ha observado que algunas aplicaciones específicas permiten utilizar material fresco, sin mayores transformaciones. Las ataduras, aplicadas en construcciones tales como refugios, viviendas, trampas y puentes, se valen de tallos de Adenocalymna marginatum y otras bignoniáceas, segmentos de las cañas de Merostachys clausseni (Poaceae), corteza de las raíces aéreas de Philodendron bipinnatifidum (Araceae), hojas de Cordyline spectabilis (Agavaceae), entre otros materiales, los cuales son utilizados inmediatamente después de ser cortados. La sección de los tallos de Adenocalymna marginatum muestra un cordón leñoso en forma de cruz de malta, con médula parenquimática (Fig. 1A). Cuando son empleados para efectuar ataduras en la construcción (Fig. 1B), previamente se los somete a torsión manual desprendiendo internamente tramos longitudinales de los lóbulos de la cruz leñosa, lo cual confiere mayor ductilidad a las lianas.

\section{Sogas, cuerdas y cordeles}

La cuerda es un conjunto de hilos que torcidos forman un solo cuerpo, más o menos flexible, grueso y largo. La soga se define como una cuerda gruesa y el cordel es una cuerda fina. Las sogas, cuerdas y cordeles que confeccionan los guaraníes constituyen piezas fundamentales en el diseño de diversos tipos de trampas y armas que utilizan. Dependiendo del tamaño de las presas que se desee capturar o ultimar, las trampas de lazo o de golpe requieren cuerdas más o menos tenaces. El material más ampliamente utilizado entre los guaraníes para este fin es la corteza de las raíces aéreas de "guembe", Philodendron bipinnatifidum (Araceae), el empleo de esta especie como textil ha sido también mencionado para otras etnias, como los guayaquíes del Paraguay (Crisci \& Gancedo, 1971) y los tobas del Chaco (Martínez Crovetto, 1964). Para capturar animales de mayor tamaño y fuerza como tapires, pecaríes o los grandes felinos se pueden utilizar sogas gruesas de "guembe" (Fig. 1C), pero son más frecuentes las cuerdas confeccionadas a partir de fibras extraídas de las vainas foliares tiernas de la palmera "pindo" Arecastrum romanzoffianum. Actualmente es común en algunas comunidades el empleo de cables de acero en el armado de trampas.

En general las cortezas que se utilizan en cordelería son sometidas al proceso de enriado (introducir en el agua por unos días para separar la fibra de otro tipo de tejidos), sin embargo la ductilidad y tenacidad de la corteza de Daphnopsis racemosa (Thymelaeaceae) permite su utilización inmediata durante una faena de caza, pesca o recolección (Fig. 1D).

La cuerda de los arcos se confecciona con corteza de las raíces aéreas de "guembe" o las fibras de las vainas foliares de la palmera "pindo", siendo este último material el predilecto por su resistencia y durabilidad.

Varios informantes han mencionado materiales que ya no se utilizan en la actualidad para confeccionar cuerdas, entre ellos la corteza de Ceiba speciosa (Bombacaceae) y las fibras de los estolones de Bromelia balansae (Bromeliaceae). Sin embargo, se ha observado entre agricultores no indígenas, inmigrantes de Brasil, la confección ocasional de sogas a partir de esta última especie. Un integrante de la parcialidad Ava Chiripa ha mencionado la confección en el pasado de cordeles a partir de las fibras de las hojas de Cordyline spectabilis (Agavaceae), las cuales se extraían manualmente después de someter las láminas al calor del fuego, sin embargo este dato no ha sido aún corroborado en la práctica.

Martínez Crovetto dejó anotaciones inéditas de sus campañas etnobotánicas llevadas a cabo hace más de cuarenta años en comunidades guaraníes de Misiones. En dichas notas, 

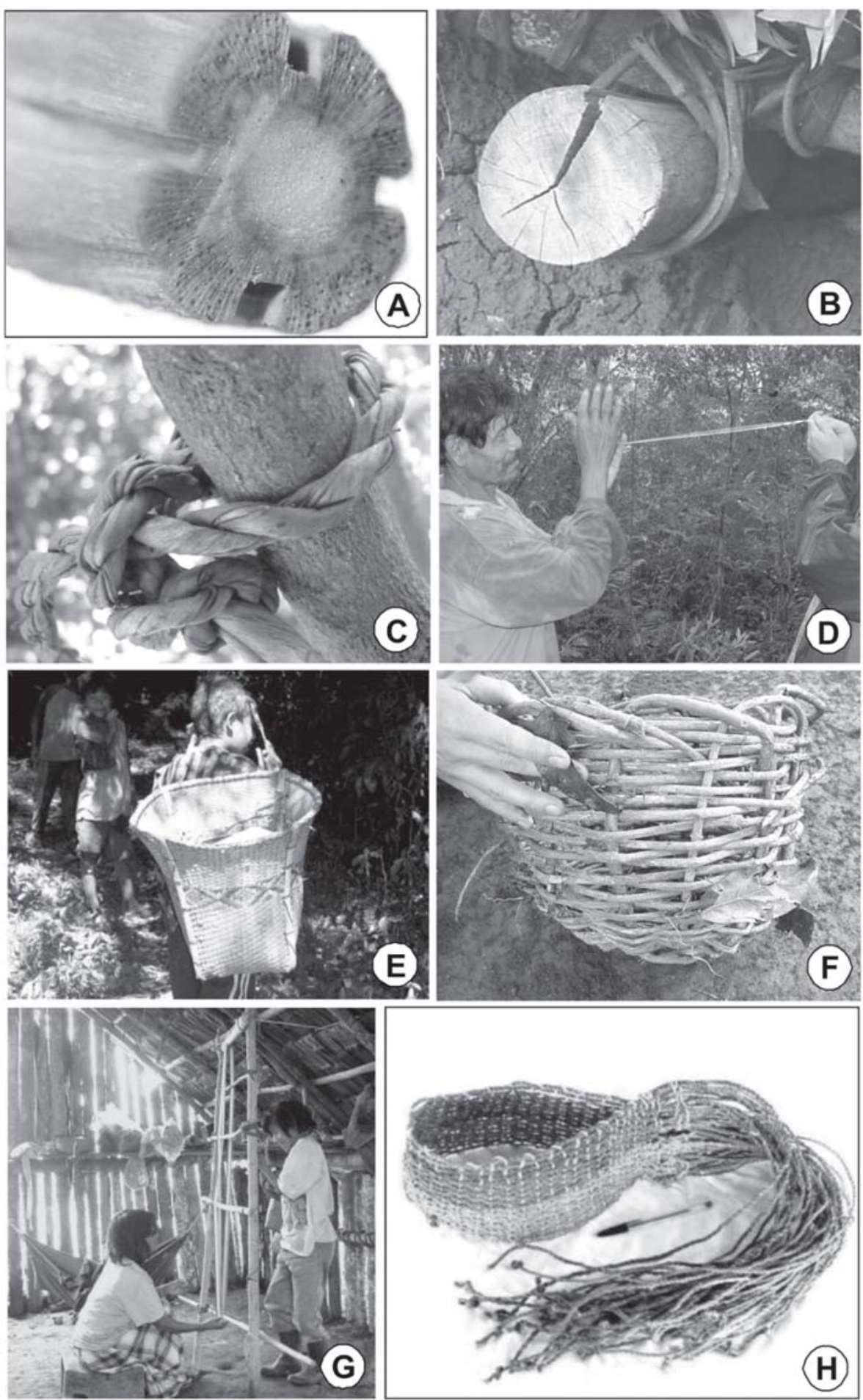

Fig. 1. A: sección del cordón leñoso del tallo de Adenocalymna marginatum (Bignoniaceae). B: atadura de construcción con la misma especie. C: trampa de lazo con soga de Philodendron bipinnatifidum (Araceae). D: confección de cordel de Daphnopsis racemosa (Thymeliaceae). E: cesta tradicional de los Mbya. F: cesta elaborada con tallos de bignoniáceas. G: telar. H: “jeguaka” ó adorno cefálico mbya confeccionado con corteza de Bastardiopsis densiflora (Malvaceae). 
que se hallan depositadas en la biblioteca del Instituto de Botánica del Nordeste, Corrientes, ha registrado el empleo de la corteza de Terminalia triflora (Combretaceae), tallos de Cyperus entrerianus (Cyperaceae) y fibras de los estolones de Pseudananas macrodontes (Bromeliaceae), en la producción de sogas y cuerdas.

Actualmente los guaraníes obtienen hilos de pescar del mercado, pero se ha mencionado en algunas comunidades el uso pretérito de cordeles confeccionados a partir de las fibras extraídas manualmente de láminas foliares de Acrocomia aculeata (Arecaceae) y Bromelia balansae (Bromeliaceae). Estos mismos hilos se empleaban para costurar prendas de vestir y bolsos de cuero (Müller, 1989). Martínez Crovetto registró el empleo de los pelos sedosos de Ceiba speciosa (Bombacaceae) para la elaboración de hilos de coser.

\section{Cestería}

En cierto tipo de cestería utilitaria tradicional se suelen utilizar materiales frescos, sin mayor procesamiento. Los Ava Chiripa se caracterizan por la confección de las cestas denominadas "yru'ague", cuyo recipiente se hace con un hemilimbo de la palmera Arecastrum romanzoffianum, que se enrosca dos veces sobre su nervio medio, de modo que los segmentos foliares de cada vuelta quedan entrecruzados, lo cual facilita el entretejido. La cuerda de suspensión consiste en una pieza entretejida aplanada hecha con la corteza de las raíces del "guembe", Philodendron bipinnatifidum (Araceae), la cual se dispone ceñida a la frente de las mujeres.

Los Mbya confeccionan sus canastos utilitarios a partir de tallos de la bambúsea “takuapi” (Merostachys clausseni), usando también la corteza de "guembe” para la confección de las cuerdas de suspensión (Fig. $1 \mathrm{E})$. Después de la floración y muerte masiva de las poblaciones de esta especie, acaecida en el año 2005, se ha podido constatar el uso de tallos de Adenocalymna marginatum (Fig. 1F) y otras Bignoniáceas para la confección de cestas utilitarias.

Los guaraníes cuentan con un amplio repertorio de diseños en cestería comercial, inclu- yendo cestos, paneras, fruteras, abanicos, sombreros, tamices, portalápices, tapizado de recipientes, entre otros. Los cestos grandes se confeccionan a partir de tallos de Merostachys clausseni (Poaceae), para los cestos pequeños y tapices se emplean tallos de Chusquea ramosissima (Poaceae); las fruteras se confeccionan a partir de dos entrenudos de Guadua trini (Poaceae). Los cordones vasculares de las raíces aéreas de Philodendron bipinnatifidum (Araceae) se suelen emplear para confeccionar sombreros y cestos pequeños. En sitios donde las bambúseas mencionadas no crecen, al menos en abundancia suficiente, se suelen utilizar tallos de otras poáceas, como Guadua angustifolia y Arundo donax.

\section{Atuendos}

Entre los guaraníes, la penetración de productos europeos eliminó la producción autóctona del tejido (Müller, 1989). Actualmente sólo se conservan algunas prendas típicas que son utilizadas por unos pocos ancianos en ocasiones ceremoniales.

En el pasado los guaraníes confeccionaban prendas no tejidas hechas con planchas de corteza (Müller, 1989). Hoy en día este uso ya ni se recuerda en las comunidades y lo más cercano a ello es el adorno cefálico que exhiben los líderes Ava Chiripá durante ciertas ceremonias y que consiste en una faja de corteza de Helietta apiculata (Rutaceae) adornada con una diadema de plumas rojas de tucán u otra ave. También constituyen técnicas similares a la elaboración de estos géneros bastos, las vendas de corteza que se aplican alrededor de los cuellos inflamados por el bocio u otras afecciones (ver último acápite).

También ha caído en desuso en las aldeas el cultivo del algodón, mencionado para los guaraníes de Misiones hace unos cuarenta años (Martínez Crovetto, 1968).

La utilización de las fibras liberianas de Urera baccifera (Urticaceae) es un recurso textil bien conocido y recordado por las personas mayores, pero solamente se ha registrado un caso de su aprovechamiento, se trata de un taparrabo que utiliza un importante diri- 
gente religioso de la parcialidad Ava Chiripa durante ciertas ceremonias anuales. El material fibroso que se obtiene de esta especie después del enriado es de consistencia algodonosa y se presta para la confección de hilos muy suaves.

Diversas malváceas poseen fibras liberianas aptas para aplicaciones textiles (Bertoni, 1903; Acosta Solís, 1952; Maiti, 1967; Luna Ercilla, 1977). En Argentina esta familia se halla representada por 198 especies, con un solo representante arbóreo (Krapovickas, 1999). Bastardiopsis densiflora es un árbol pionero, común en selvas secundarias y antiguos sectores de entresaca. Para la confección de ciertas prendas ceremoniales, los Mbya, ocasionalmente utilizan la corteza de este árbol. En primer lugar los jóvenes seleccionan los ejemplares adecuados, árboles vigorosos con troncos de cerca de $20 \mathrm{~cm}$ de diámetro (no muy pequeños ni muy añosos). Al tratarse de una especie pionera se pueden hallar abundantes ejemplares en sucesiones secundarias posteriores a la roza y quema, y los bordes de selva. Una vez seleccionados los ejemplares, se abren muescas con machete en la base del fuste hasta llegar a la zona cambial, a partir de ellas se arrancan largas cintas de corteza, las que se desprenden a la altura de las primeras ramificaciones, a unos 5 ó $6 \mathrm{~m}$ de altura. Luego se separa y desecha la corteza externa o peridermis, y las tiras de corteza interna resultantes son atadas en manojos y sometidas al enriado. Un estudio anatómico de esta corteza revela la presencia de abundantes paquetes de fibras y canales de mucílago inmersos en un tejido parenquimático (Keller \& al., 2003). Mediante el enriado se consigue aislar el tejido fibroso eliminando los demás componentes. Luego de varios días se retiran los manojos del curso de agua y se dejan secar al sol durante un día completo. A partir de esta materia prima, los ancianos arman los cordeles retorciendo dos paquetes de bandas de fibra y enroscando ambas unidades entre sí. Se observó que con el fin de darle firmeza al producto, atan el extremo inicial del cordel al dedo mayor de un pie y lo mantienen firme mientras retuercen las bandas de corteza y trenzan las dos unidades mencionadas. A me- dida que los paquetes que conforman cada unidad se aguzan, ellos agregan más bandas de fibra, lo que permite prolongar indefinidamente la longitud del cordel. Con el fin de que el producto cuente con colores diferentes, se tiñe parte de los cordeles ya elaborados con un colorante obtenido de la corteza de Trichilia catigua (Meliaceae). Luego se efectúa el tejido mediante el empleo de un improvisado telar vertical (Fig. 1G). El resultado de esta labor es un adorno cefálico como la que se observa en la Figura $1 \mathrm{H}$.

\section{Hamacas}

Los guaraníes confeccionan hamacas para descansar durante las siestas o bien como sitio de pernoctación de niños pequeños. Estas hamacas tradicionalmente se confeccionaban en telares horizontales, donde se tejían tramas compactas de hilos de algodón, de "pyno" (Urera baccifera), o cordeles de corteza. Actualmente se elaboran manualmente hamacas tipo redes (con entramado laxo) empleando para ello cuerdas de las fibras de las vainas foliares de Arecastrum romanzoffianum (Arecaceae), corteza de raíces de Philodendron bipinnatifidum (Araceae) y chalas de la mazorca de maíz trenzadas.

\section{Otras aplicaciones}

Las plantas textiles también tienen aplicación en la medicina y la magia de los guaraníes. Con cintas de la corteza de Ficus luschnatiana (Moraceae) se elaboran vendas de corteza, que se ciñen en torno al cuello de la persona que padece de bocio, a la pierna de quien padece de retención de líquidos u otra afección inflamatoria.

Durante los partidos de fútbol que se llevan a cabo en las comunidades, algunos espectadores portan secretamente pequeños cordeles de la corteza de Sida rhombifolia y otras malváceas similares, los cuales en el momento de una acción ofensiva (tiros libres, ejecución de penales) tensan repentinamente hasta su ruptura, como procedimiento mágico para entorpecer a los oponentes del equipo con el cual simpatizan. 


\section{Conclusiones y Recomendaciones}

La incorporación de vestimentas, abrigos, hilos de pescar, cuerdas, recipientes y otros elementos propios de la sociedad global ha erosionado tempranamente el empleo de plantas textiles entre los guaraníes de Misiones. Esta tendencia se mantiene en la actualidad y diversas especies y aplicaciones textiles que se mencionaban hace pocas décadas, son actualmente desconocidas.

Por otra parte, el conocimiento de muchas técnicas de uso de plantas textiles es actualmente patrimonio exclusivo de los ancianos guaraníes. Es urgente tomar medidas que permitan recuperar y consolidar dichos saberes, proceso en el cual la Etnobotánica juega un rol fundamental.

\section{Agradecimientos}

A los integrantes de las comunidades guaraníes donde se llevó a cabo el estudio. Al Ing. Antonio Krapovickas por poner a mi disposición las notas de campo inéditas de Martínez Crovetto. A los especialistas del Instituto de Botánica del Nordeste (UNNECONICET) que han colaborado con la identificación del material de herbario.

\section{Bibliografía}

BERTONI, M. S. 1911. Una nueva planta de fibras: Pavonia restiaria Bertoni. Anales Ci. Parag. 9 (1): 1-20.
CRISCI, J. V. \& O. GANCEDO. 1971. Sistemática y etnobotánica del Guembé (Philodendron bipinnatifidum) una importante arácea sudamericana. Rev. Mus. La Plata., Secc. Bot. XI (65): 285-302.

KELLER, H. A, A. KRAPOVICKAS, D. MIRANDA, L. GRANCE, A. BÖHREN \& D. CABANNE. 2003. Bastardiopsis densiflora (Hook. et Arn.) Hassl. (Malvaceae): Un antiguo árbol textil de los Guaraníes de Misiones, Argentina. XIV Reunión de Comunicaciones Científicas y Técnicas 2003. Facultad de Ciencias Agrarias - UNNE, Corrientes. http://agr.unne.edu.ar/Extension/Res2003/ Botanica/Botan-003.pdf

KRAPOVICKAS, A. 1999. Malvaceae. En: F. Zuloaga \& O. Morrone. Catálogo de las plantas vasculares de la República Argentina II. Monogr. Syst. Bot. Missouri Bot. Gard. 813-844.

LANGE, J. 1966. Primitive Plantenavne og deres gruppering efter motiver. J. Jøgensen \& Co Bogtrykkeri. København. 157 pp.

LUNA ERCILLA, C. A. 1977. Plantas textiles indígenas. Enciclopedia Argentina de Agricultura y Jardinería. Segunda edición, Tomo II, fascículo 18-2. ACME, Buenos Aires. 72 pp.

MAITI, R. K. 1969. Hibiscus vitifolius, a new fiber crop. Econ. Bot. 23(2): 141-147.

MARTÍNEZ CROVETTO, R. 1964. Estudios etnobotánicos I. Nombres de plantas y su utilidad según los indios tobas del este del Chaco. Bonplandia 1(4): 279-333.

—. 1968. Notas sobre la agricultura de los indios guaraníes de Misiones. Etnobiológica 10: 1-11.

MELIÁ, B. 2005. Cuarenta años de presencia guaraní en el Suplemento Antropológico. Suplemento Antropológico 40(1): 1-11.

MÜLLER, F. 1989. Etnografía de los Guaraní del Alto Paraná. Socieatis Verbi Divini, Rosario. 132 pp.

NIMUENDAJU (UNKEL), C. 1914. Die Sagen von der Erschaffung und Vernichtung der Welt als Grundlagen der Religion der Apapocúva-Guaraní. Zeitschrift für Ethnologie XLVI: 284-403.

Original recibido el 18 de mayo de 2009; aceptado el 20 de junio de 2009. 
Apéndice: Lista de especies textiles guaraníes, se detalla el nombre guaraní, el uso, la parte usada, la vigencia del uso (A. actual; E: esporádico; P: pretérito) y un ejemplar testigo. Código de coleccionistas: K: Keller o Keller et al.; T: Tressens et al. Departamentos: C: Concepción; Ca: Candelaria; E: Eldorado; G: Guaraní; I: Iguazú, L: Libertador

General San Martín; M: Montecarlo; S: San Pedro.

\begin{tabular}{|c|c|c|c|c|c|}
\hline ESPECIE & NOMBRE & USO & PARTE USADA & VIGENCIA & TESTIGO \\
\hline \multicolumn{6}{|l|}{ DICOTYLEDONEAE } \\
\hline \multicolumn{6}{|l|}{ Bignoniaceae } \\
\hline $\begin{array}{l}\text { Adenocalymna marginatum } \\
\text { (Cham.) DC. }\end{array}$ & ychypo u & $\begin{array}{l}\text { ataduras rústicas, } \\
\text { cestería }\end{array}$ & tallos & A & K 3467 (S) \\
\hline $\begin{array}{l}\text { Mansoa difficilis (Cham.) Bureau } \\
\text { \& K. Schum. }\end{array}$ & ychypo ũ & $\begin{array}{l}\text { ataduras rústicas, } \\
\text { cestería }\end{array}$ & tallos & A & T $4609(G)$ \\
\hline $\begin{array}{l}\text { Pyrostegia venusta (Ker Gawl.) } \\
\text { Miers }\end{array}$ & ychypo ete & $\begin{array}{l}\text { ataduras rústicas, } \\
\text { cestería }\end{array}$ & tallos & A & K 1359 (S) \\
\hline \multicolumn{6}{|l|}{ Bombacaceae } \\
\hline $\begin{array}{l}\text { Ceiba speciosa (A.St.-Hil., Juss. } \\
\text { \& Cambess.) Ravenna }\end{array}$ & yvi & $\begin{array}{l}\text { cordeles, soga para } \\
\text { hamacas (1), hilos (2) }\end{array}$ & $\begin{array}{l}\text { fibras liberianas (1), } \\
\text { fibras de los frutos } \\
\text { (2) }\end{array}$ & $\mathrm{P}$ & T $5632(G)$ \\
\hline \multicolumn{6}{|l|}{ Boraginaceae } \\
\hline Cordia trichotoma (Vell.) Arrab. & apeteryvi & $\begin{array}{l}\text { probablemente usado } \\
\text { en el pasado como } \\
\text { textil }\end{array}$ & fibras liberianas & $\mathrm{P}$ & K 1666 (E) \\
\hline Patagonula americana L. & guajayvi & $\begin{array}{l}\text { probablemente usado } \\
\text { en el pasado como } \\
\text { textil }\end{array}$ & fibras liberianas & $\mathrm{P}$ & T $4744(G)$ \\
\hline \multicolumn{6}{|l|}{ Combretaceae } \\
\hline Terminalia australis Cambess. & chara & cuerdas & fibras liberianas & $\mathrm{P}$ & T $5501(G)$ \\
\hline \multicolumn{6}{|l|}{ Malvaceae } \\
\hline $\begin{array}{l}\text { Bastardiopsis densiflora (Hook. } \\
\text { \& Arn.) Hassl. }\end{array}$ & mandyjurã & $\begin{array}{l}\text { indumentaria } \\
\text { ceremonial, hamacas }\end{array}$ & fibras liberianas & $\mathrm{E}$ & K $1944(G)$ \\
\hline Sida rhombifolia L. & typycha & magia & corteza & A & T $4714(\mathrm{G})$ \\
\hline Sida spinosa L. & typycha & magia & corteza & A & K $837(\mathrm{M})$ \\
\hline $\begin{array}{l}\text { Malvastrum coromandelianum } \\
\text { (L.) Garcke }\end{array}$ & typicha ũ & magia & corteza & A & K 5665 (C) \\
\hline \multicolumn{6}{|l|}{ Moraceae } \\
\hline Ficus luschnathiana (Miq.) Miq. & guapoy & ropas duras, vendas & corteza & A & T $4911(G)$ \\
\hline \multicolumn{6}{|l|}{ Rutaceae } \\
\hline Helietta apiculata Benth. & yvyra ovy & $\begin{array}{l}\text { indumentaria } \\
\text { ceremonial (vinchas) }\end{array}$ & corteza & $\mathrm{E}$ & K 1369 (S) \\
\hline \multicolumn{6}{|l|}{ Thymelaeaceae } \\
\hline Daphnopsis racemosa Griseb. & guajayvi chî & $\begin{array}{l}\text { ataduras rústicas, } \\
\text { cuerdas }\end{array}$ & corteza & A & K 3519 (G) \\
\hline \multicolumn{6}{|l|}{ Urticaceae } \\
\hline Urera bacciferaWedd. & pyno & $\begin{array}{l}\text { atuendos, mantas, } \\
\text { hamacas, redes de } \\
\text { pesca }\end{array}$ & fibras liberianas & $\mathrm{P}$ & K 2958 (L) \\
\hline
\end{tabular}




\begin{tabular}{|c|c|c|c|c|c|}
\hline ESPECIE & NOMBRE & USO & PARTE USADA & VIGENCIA & TESTIGO \\
\hline \multicolumn{6}{|l|}{ MONOCOTYLEDONEAE } \\
\hline \multicolumn{6}{|l|}{ Agavaceae } \\
\hline $\begin{array}{l}\text { Cordyline spectabilis Kunth \& } \\
\text { Bouché }\end{array}$ & yvara & $\begin{array}{l}\text { ataduras de poca } \\
\text { duración, cordeles }\end{array}$ & $\begin{array}{l}\text { hojas, fibras } \\
\text { foliares }\end{array}$ & A & K 1352 (S) \\
\hline \multicolumn{6}{|l|}{ Araceae } \\
\hline $\begin{array}{l}\text { Philodendron bipinnatifidum } \\
\text { Schott }\end{array}$ & guembe & $\begin{array}{l}\text { ataduras rústicas, } \\
\text { cuerdas, cestería, } \\
\text { hamacas (1) } \\
\text { sombreros, cestos } \\
\text { pequeños (2) }\end{array}$ & $\begin{array}{l}\text { corteza de las } \\
\text { raíces aéreas (1), } \\
\text { cordones } \\
\text { vasculares de las } \\
\text { raíces aéreas (2) }\end{array}$ & A & K $1432(\mathrm{M})$ \\
\hline \multicolumn{6}{|l|}{ Arecaceae } \\
\hline $\begin{array}{l}\text { Acrocomia aculeata Lodd. ex } \\
\text { Mart. }\end{array}$ & mbokaja & $\begin{array}{l}\text { hilos para pescar y } \\
\text { para costurar }\end{array}$ & fibras de las hojas & $\mathrm{P}$ & K 1676 (E) \\
\hline $\begin{array}{l}\text { Arecastrum romanzoffianum } \\
\text { (Cham.) Becc. }\end{array}$ & pindo & $\begin{array}{l}\text { ataduras rústicas, } \\
\text { cestos utilitarios (1), } \\
\text { cuerdas de arcos, } \\
\text { ataduras de tambores, } \\
\text { sogas y cordeles para } \\
\text { trampas, hamacas (2) }\end{array}$ & $\begin{array}{l}\text { hojas (1), fibras } \\
\text { de las vainas } \\
\text { foliares tiernas (2) }\end{array}$ & A & K 3431 (S) \\
\hline $\begin{array}{l}\text { Butia paraguayensis (Barb. } \\
\text { Rodr.) L.H.Bailey }\end{array}$ & jatay & $\begin{array}{l}\text { ataduras de poca } \\
\text { duración }\end{array}$ & hojas & $\mathrm{P}$ & K 4572 (Ca) \\
\hline Euterpe edulis Mart. & jejy & ataduras rústicas & hojas & A & K $1642(G)$ \\
\hline \multicolumn{6}{|l|}{ Bromeliaceae } \\
\hline Bromelia balansae Mez & karaguata & $\begin{array}{l}\text { sogas (1), hilos para } \\
\text { pescar y costurar (2) }\end{array}$ & $\begin{array}{l}\text { fibras de los } \\
\text { estolones (1), } \\
\text { fibras foliares (2) }\end{array}$ & $\mathrm{P}$ & K 1502 (I) \\
\hline $\begin{array}{l}\text { Pseudananas macrodontes } \\
\text { (Morr.) Harms }\end{array}$ & karaguata re'y & sogas, cordeles & $\begin{array}{l}\text { fibras de las hojas } \\
\text { y rizomas }\end{array}$ & $\mathrm{P}$ & K $1412(\mathrm{Ca})$ \\
\hline \multicolumn{6}{|l|}{ Cyperaceae } \\
\hline Cyperus entrerianus Boeck. & piri & sogas & tallos & $\mathrm{P}$ & --- \\
\hline \multicolumn{6}{|l|}{ Poaceae } \\
\hline Arundo donax L. & takuachî & cestería & tallos & $\mathrm{E}$ & K 1042 (E) \\
\hline Chusquea ramosissima Lindm. & takuarembo & cestería & tallos & A & K 1235 (M) \\
\hline Guadua angustifolia Kunth & takuaruchu & cestería & tallos & $\mathrm{E}$ & K $1681(\mathrm{E})$ \\
\hline Guadua trini (Nees) Rupretch & pekuru & cestería & tallos & A & K 1375 (I) \\
\hline Merostachys clausseni Munro & takuapi & $\begin{array}{l}\text { ataduras rústicas, } \\
\text { cestería }\end{array}$ & tallos & A & K 2828 (S) \\
\hline Zea mays L. & avachi & cuerdas para hamacas & $\begin{array}{l}\text { chala de las } \\
\text { mazorcas }\end{array}$ & A & K 671 (S) \\
\hline
\end{tabular}


BONPLANDIA 18(1). 2009 